\title{
Fluorescence Lifetime Imaging Microscopy for the Monitoring of Green Fluorescent Protein-Tagged Androgen Receptors in Living Cells
}

\author{
Rina Miyake, ${ }^{a}$ Tomohiro Uchimura, ${ }^{*, a, b, c} \mathrm{Xu} \mathrm{Li},{ }^{a, d}$ and Totaro Imasaka ${ }^{a, b}$ \\ ${ }^{a}$ Department of Applied Chemistry, Graduate School of Engineering, Kyushu University; ${ }^{b}$ Department of \\ Translational Research Center, Center for Future Chemistry, Kyusyu University; Nishi-ku, Fukuoka 819-0395, Japan: \\ ${ }^{c}$ Department of Materials Science and Engineering, Graduate School of Engineering, University of Fukui; Bunkyo, \\ Fukui 910-8507, Japan: and ${ }^{d}$ Department of Orthopedic Surgery, First Affiliated Hospital, China Medical University; \\ Heping District, Shenyang 110001, China.
}

Received May 9, 2012; accepted October 4, 2012

\begin{abstract}
Fluorescence lifetime imaging microscopy (FLIM) was used to monitor the interaction between androgen receptor (AR) tagging of a green fluorescent protein (GFP) and the ligands in living cells. The fluorescence lifetime of the AR-GFP without ligands was $c a .3 .1 \mathrm{~ns}$, which was reduced to $c a$. $2.5 \mathrm{~ns}$ after treatment with agonist $5 \alpha$-dihydrotestosterone. On the other hand, the fluorescence lifetime of AR-GFP was not changed after treatment with antagonist hydroxyflutamide. The reaction kinetics was simulated in the present study, and the obtained results indicated the possibility of the presence of an intermediate complex during the reaction. FLIM can be used to record the ratio of the AR as it reacts with an agonist, and, therefore, it is useful for acquiring information concerning the interaction between AR and ligands in living cells.
\end{abstract}

Key words androgen receptor; $5 \alpha$-dihydrotestosterone; hydroxyflutamide; fluorescence lifetime imaging microscopy; green fluorescent protein

Fluorescence intensity imaging microscopy has been widely used in cytology and histology. However, fluorescence intensity images cannot quantitatively be compared with each other, since fluorescence intensity is deduced as a relative value. On the other hand, fluorescence lifetime is measured as an absolute, and is influenced by the characteristics of a fluorophore's microenvironment, such as temperature, viscosity, and $\mathrm{pH}$. Therefore, it is possible to use fluorescence lifetime imaging microscopy (FLIM) to evaluate biological phenomena in living cells. ${ }^{1,2)}$ We have recently developed a fluorescence lifetime imaging microscope that consists of a picosecond dye laser and a time-gated intensified charge-coupled device (ICCD) camera. ${ }^{3,4)}$

In this study, the FLIM system was applied to the monitoring of an androgen receptor (AR) with tagging green fluorescent protein (GFP) in living cells. AR is one of the steroid hormone receptors present in the cytoplasm without ligands, and it is subsequently translocated into the nucleus after binding. ${ }^{5)}$ Until now, the location of AR in living cells has been observed primarily through the use of fluorescence intensity images. ${ }^{6-8)}$ We confirmed the intracellular location of ARGFP after treatment with $5 \alpha$-dihydrotestosterone (DHT) and a hydroxyflutamide (OH-FLU) by using the FLIM system. Moreover, the results of the dose-dependent experiment were also simulated in the present study.

\section{Experimental}

Cell Culture and Transfection of AR-GFP COS-7 cells were obtained from the Japanese Collection of Research Bioresources, and were maintained in Dulbecco's minimal essential medium (DMEM; Invitrogen, U.S.A.) supplemented with $10 \%$ fetal bovine serum (FBS; Gibco, U.S.A.) and AntibioticAntimycotic at $37^{\circ} \mathrm{C}$ and $5 \% \mathrm{CO}_{2}$.

The AR-GFP plasmids (pEGFP-N2) were kindly provided by H. Iwamoto (Kyushu University). The cells were cultured

The authors declare no conflict of interest. in $35 \mathrm{~mm}$ glass-bottom dishes (Mat-Tek) $\left(5 \times 10^{4}\right.$ cells/dish) on the day before transfection. The AR-GFP plasmids and transfection reagent (Superfect transfection reagent, Invitrogen) were added to $100 \mu \mathrm{L}$ DMEM without FBS and Antibiotic-Antimycotic in a tube. DMEM $(600-\mu \mathrm{L})$ containing $10 \%$ FBS and Antibiotic-Antimycotic was added to the mixture in the tube, then the entire mixture was transferred to a dish. After incubation at $37^{\circ} \mathrm{C}$ and $5 \% \mathrm{CO}_{2}$ for $2-3 \mathrm{~h}$, the mixture was washed by PBS. The cells were incubated for $16-20 \mathrm{~h}$ in the absence of ligands, and then $20 \mu \mathrm{L}$ of DHT or OH-FLU (Wako, Japan) were added to the cells in given concentrations.

FLIM Measurements The FLIM system has been reported in detail elsewhere ${ }^{3,4)}$ and is described only briefly here. The picosecond dye laser (Twinstars Mini, Ishikawa Iron Works, Japan) pumped by the third harmonic emission of a Nd:YAG laser (Continuum, Minilite I) was used to excite the cells on the inverted microscope (Nikon, TE-2000U). The laser dye used in this study was Coumarin $102(480 \mathrm{~nm})$ dissolved in methanol. The fluorescence was detected with a gated-ICCD camera (Nikon, C-7300-10-12). From the obtained images, a fluorescence lifetime image was constructed using a homemade program written by LabVIEW (National Instruments, Japan). The equations for kinetics were calculated using the 4th-order Runge-Kutta method with a LabVIEW program.

\section{Results and Discussion}

Fluorescence Lifetimes before and after Ligand Treatment Figure 1 shows the fluorescence lifetime images before and after DHT treatment. In the absence of DHT, the green fluorescence of AR-GFP was observed from the cytoplasm, and was subsequently observed from the fields of both the cytoplasm and the nucleus upon DHT treatment. The change in the fluorescence lifetime of AR-GFP for each incubation time is shown in Fig. 2. The fluorescence lifetime without DHT was $c a$. $3.1 \mathrm{~ns}$, which was reduced to $c a$. $2.5 \mathrm{~ns}$ after the addition of DHT. Note that during migration, no distinctive 


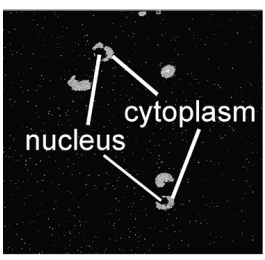

DHT(-)

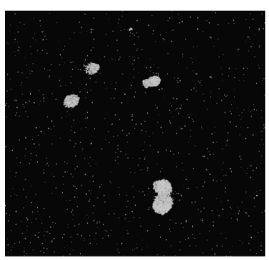

$\mathrm{DHT}(+) 180 \mathrm{~min}$
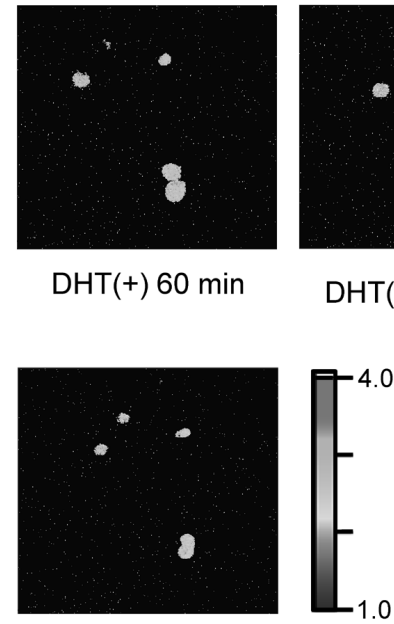

$\mathrm{DHT}(+) 240 \mathrm{~min} \tau / \mathrm{ns}$
$\mathrm{DHT}(+) 60 \mathrm{~min}$

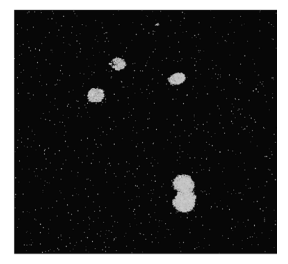

$\mathrm{DHT}(+) 120 \min$
Fig. 1. Fluorescence Lifetime Images of COS-7 Following Treatment with $1 \times 10^{-7} \mathrm{M}$ of DHT

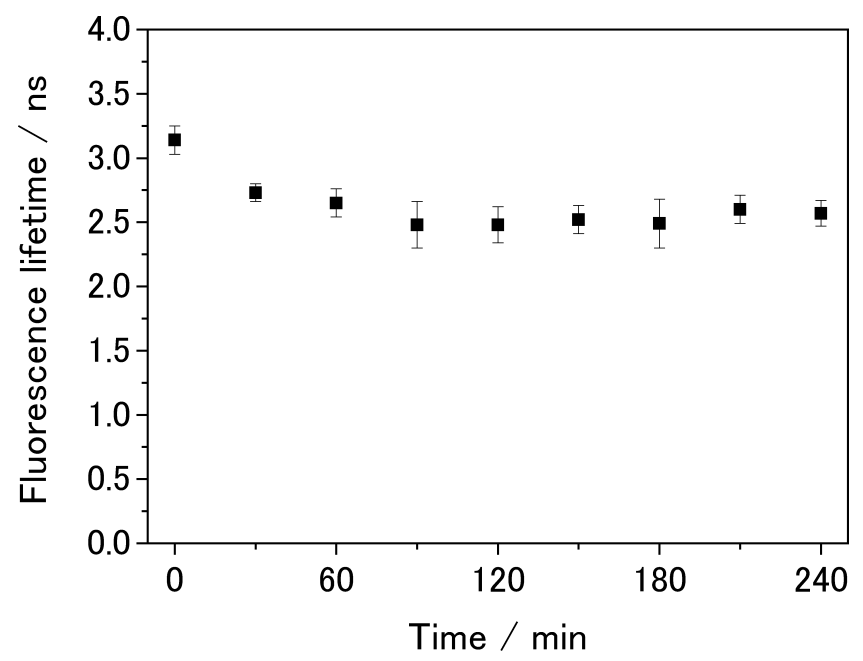

Fig. 2. The Dependence of the Fluorescence Lifetimes of COS-7 on the Incubation Times Following Treatment with $1 \times 10^{-7} \mathrm{M}$ of DHT $(n=13-24)$

difference in the fluorescence lifetime between the cytoplasm and the nucleus was found, as shown in Fig. 1. Thus, the decrease in the fluorescence lifetime was induced by the change in the microenvironment around the fluorophore derived from the binding of DHT to AR-GFP. The conformational change of AR reportedly occurs after binding with agonists, but not antagonists. ${ }^{9)}$ We also measured the FLIM images of ARGFP treated with OH-FLU as an antagonist (Fig. S1 of the Supplemental information). Similar to DHT treatment, the fluorescence was first observed from the cytoplasm, followed by the fields of both the cytoplasm and the nucleus after the addition of OH-FLU. However, the fluorescence lifetime did not decrease with OH-FLU treatment, as shown in Fig. S2 (Supplemental information). Therefore, the decrease in the fluorescence lifetime was probably caused by the conformational change of AR-GFP after binding with DHT, which was followed by the change in electrostatic, steric and/or hydrophobic interactions between the chromophore and the amino acid residues of the polypeptide chain around the chromophore. ${ }^{10)}$

Kinetics Figure 3 shows the time courses of the fluorescence lifetimes of AR-GFP combined with DHT at different
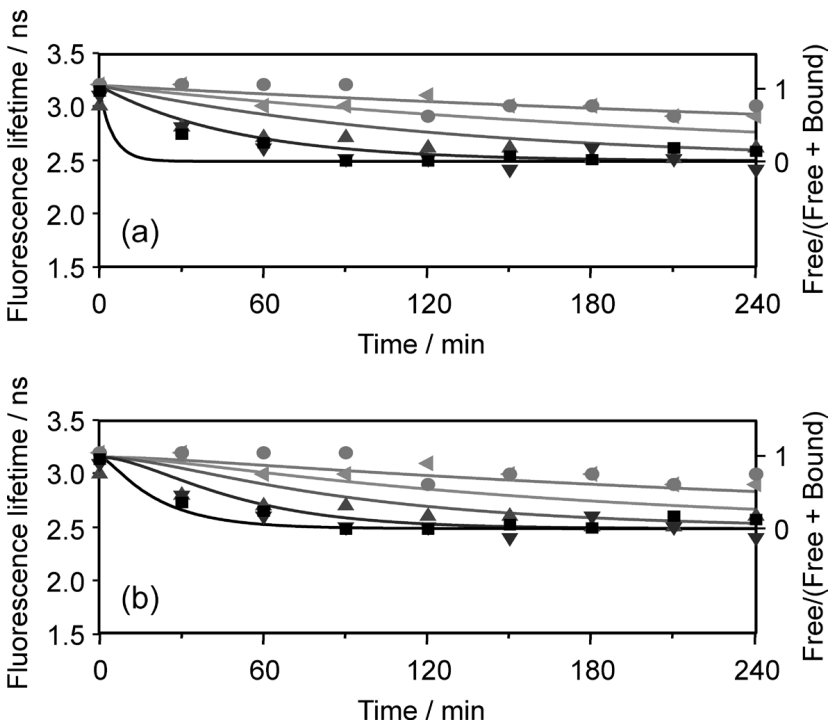

Fig. 3. Dependence of the Fluorescence Lifetimes of COS-7 on the Incubation Times Following Treatment with DHT in Concentrations Ranging from $10^{-7}$ to $10^{-9} \mathrm{M}$. $[D H T]$; (O) $1 \times 10^{-9} \mathrm{M},(\varangle) 2 \times 10^{-9} \mathrm{M}$,

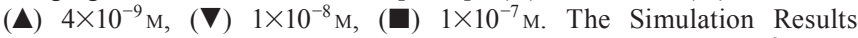
of $[A R] /[A R]+[A R-D H T]$ Are Also Indicated. $[A R]_{0} ; 1 \times 10^{-8} \mathrm{M}$. (a) $k_{1}=2 \times 10^{6} \mathrm{M}^{-1} \mathrm{~min}^{-1}$. (b) $k_{2}$ and $k_{3}$ Are $3 \times 10^{6} \mathrm{M}^{-1} \mathrm{~min}^{-1}$ and $5 \times 10^{-2} \mathrm{~min}^{-1}$, Respectively

concentrations. As shown in this figure, the fluorescence lifetime was reduced to $c a$. $2.5 \mathrm{~ns}$ at higher concentrations, which seems to be the lowest value obtained under these experimental conditions. The obtained plots were temporarily fitted with mono-exponential curves, and the half-lives of the curves were calculated to be $c a$. 30,40 , and $67 \mathrm{~min}$ at concentrations of $1 \times 10^{-7}, 1 \times 10^{-8}$, and $4 \times 10^{-9} \mathrm{M}$, respectively. If the change in the fluorescence lifetime is directly proportional to the ratio of $[A R]$ and $[A R]+[A R-D H T]$ in the cell, where $[A R]$ and $[A R-D H T]$ are the concentrations of the free $\mathrm{AR}$ in the cell and that of AR after DHT binding, respectively, the fluorescence lifetime will obey the following equation:

$$
\tau=3.1 \times \frac{[A R]}{[A R]+[A R-D H T]}+2.5 \times \frac{[A R-D H T]}{[A R]+[A R-D H T]}
$$

The ratio is indicated at the right-hand side of the ordinate in Fig. 3.

In the present study, the decrease in the fluorescence lifetime of AR-GFP after the addition of DHT was simulated. To begin with, we made assumptions to simplify the calculation: (i) the rate of membrane permeation is fast, and, hence, it was ignored; and, (ii) the concentration of DHT in culture media does not change during the reaction, and the concentration inside the cells is identical to that outside the cells, i.e., in the culture media. The binding reaction is given by the following scheme:

$$
\mathrm{AR}+\mathrm{DHT} \underset{k_{-1}}{\stackrel{k_{1}}{\rightleftarrows}} \mathrm{AR}-\mathrm{DHT}
$$

In the present study, the reverse reaction, i.e., the dissociation process, was ignored in order to simplify the simulation, although the dissociation constant, typically $10^{-7}-10^{-10} \mathrm{M}$, was reported..$^{11-13)}$ The resultant rate equation for the binding 
reaction can be expressed as follows:

$$
\frac{\mathrm{d}[A R]}{\mathrm{d} t}=-k_{1}[A R][D H T]
$$

where $[D H T]$ is the concentration of the free DHT in the cell. From the aforementioned assumption (ii), $[D H T]$ is constant, which means the above reaction can be calculated as a pseudo-first-order reaction. Figure 3 a shows an example of the time courses of $[A R]$ after the addition of DHT. Though several fittings were performed, we could not fit the decay curves calculated from the single-step bimolecular interaction expressed by reaction (2) to the obtained fluorescence lifetimes. In that case, the following reaction mechanism should be considered, where a stabilized complex is formed via a labile intermediate (AR-DHT)*

$$
\mathrm{AR}+\mathrm{DHT} \underset{k_{-2}}{\stackrel{k_{2}}{\rightleftarrows}}(\mathrm{AR}-\mathrm{DHT})^{*} \underset{k_{-3}}{\stackrel{k_{3}}{\rightleftarrows}} \mathrm{AR}-\mathrm{DHT}
$$

Several groups have reported the 2-step reaction mechanism shown above. ${ }^{11,12)}$ In the present study, the reverse reactions were also ignored to simplify the calculation, hence, the rate equations for the 2-step reaction were as follows:

$$
\begin{gathered}
\frac{\mathrm{d}[A R]}{\mathrm{d} t}=-k_{2}[A R][D H T] \\
\frac{\mathrm{d}\left[(A R-D H T)^{*}\right]}{\mathrm{d} t}=k_{2}[A R][D H T]-k_{3}\left[(A R-D H T)^{*}\right]
\end{gathered}
$$

Figure $3 b$ shows an example of the time course of $[A R]$. The values of the half-life were $20,46,80 \mathrm{~min}$ at $1 \times 10^{-7}, 1 \times 10^{-8}$, and $4 \times 10^{-9} \mathrm{M}$, respectively, which were nearly consistent with the experimental results. This suggests the presence of the intermediate and the product after the conformational change, though future studies are required to elucidate the conclusion including the rate of membrane permeation and dissociation of the intermediate and/or complex.

\section{Conclusion}

Fluorescence lifetime images of AR-GFP associated with ligands, DHT and OH-FLU, were measured. The fluorescence lifetime decreased only after DFT treatment, which cannot be attributed to intracellular localization, but instead to a change in the microenvironment. Moreover, the kinetics of the binding with AR-GFP and DHT was verified, and the obtained results indicated that the binding reaction included a 2-step process. The agonist-mediated AR-GFP can be monitored by FLIM, which cannot be achieved by observation of the fluorescence intensity. Therefore, FLIM is useful for evaluation of the binding of ligands to the AR-GFP in living cells.

Acknowledgments The present work was supported by a Grant-in-Aid for the Global COE Program, "Science for Future Molecular Systems" from the Ministry of Education, Culture, Sports, Science and Technology of Japan. T.U. acknowledges support by Kyushu University Interdisciplinary Programs in Education and Projects in Research Development. We are indebted to H. Iwamoto of Kyushu University for providing the AR-GFP plasmids.

\section{References}

1) Wallrabe H., Periasamy A., Curr. Opin. Biotechnol., 16, 19-27 (2005).

2) Calleja V., Ameer-Beg S. M., Vojnovic B., Woscholski R., Downward J., Larijani B., Biochem. J., 372, 33-40 (2003).

3) Uchimura T., Kawanabe S., Maeda Y., Imasaka T., Anal. Sci., 22, 1291-1295 (2006).

4) Li X., Uchimura T., Kawanabe S., Imasaka T., Anal. Biochem., 367, 219-224 (2007).

5) Guiochon-Mantel A., Delabre K., Lescop P., Milgrom E., J. Steroid Biochem. Mol. Biol., 56 (1-6 Spec. No.), 3-9 (1996).

6) Tyagi R. K., Lavrovsky Y., Ahn S. C., Song C. S., Chatterjee B., Roy A. K., Mol. Endocrinol., 14, 1162-1174 (2000).

7) Saitoh M., Takayanagi R., Goto K., Fukamizu A., Tomura A., Yanase T., Nawata H., Mol. Endocrinol., 16, 694-706 (2002).

8) Nakauchi H., Matsuda K.-I., Ochiai I., Kawauchi A., Mizutani Y., Miki T., Kawata M., J. Histochem. Cytochem., 55, 535-544 (2007).

9) Kallio P. J., Jänne O. A., Palvimo J. J., Endocrinology, 134, 998 1001 (1994).

10) Nakabayashi T., Ohta N., Bunseki Kagaku, 58, 473-485 (2009).

11) Pratt W. B., Kaine J. L., Pratt D. V., J. Biol. Chem., 250, 4584-4591 (1975).

12) Mayeux P., Felix J. M., Billat C., Jacquot R., Biochim. Biophys. Acta, 763, 205-211 (1983).

13) Georget V., Térouanne B., Lumbroso S., Nicolas J.-C., Sultan C., J. Clin. Endocrinol. Metab., 83, 3597-3603 (1998). 